\title{
Descripción del Somatotipo y Cualidades Físicas de Varones Surfistas Experimentados Chilenos
}

\author{
Description of Somatotype and Physical Qualities of Experienced Chilean Men Surfers
}

\author{
María Ignacia Valdés V.* \& Rodrigo Guzmán-Venegas*
}

VALDÉS, V. M. I. \& GUZMÁN-VENEGAS, R. Descripción del somatotipo y cualidades físicas de varones surfistas experimentados chilenos. Int. J. Morphol., 34(1):23-28, 2016.

RESUMEN: El surf es un deporte que ha incrementado su número de adeptos en Chile. Sin embargo, existen pocos estudios que describan las características antropométricas y físicas de estos deportistas en el medio nacional. Este estudio observacional descriptivo transversal, tuvo por objetivo describir las características antropométricas y físicas de surfistas experimentados chilenos. En 15 surfistas

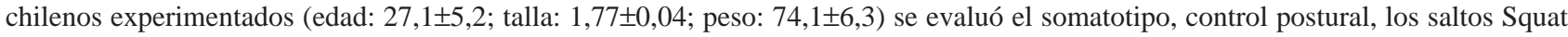
Jump (SJ) y Counter Movement Jump (CMJ), y el consumo máximo de oxígeno ( $\mathrm{VO}_{2}$ Max.). El somatotipo promedio de la muestra fue endomorfo $(2,7 \pm 0,5)$, mesomorfo $(4,6 \pm 0,7)$ y ectomorfo $(2,3 \pm 0,6)$. En la prueba de estabilidad postural se obtuvo un promedio de la velocidad y de área de desplazamiento del centro de presión de 7,2 $\pm 1,3 \mathrm{~mm} / \mathrm{seg}$ y $68,1 \pm 18,4 \mathrm{~mm}^{2}$ respectivamente. La altura promedio

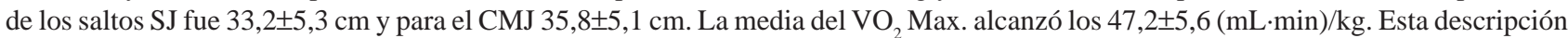
reveló que el somatotipo de la muestra fue mesomórfico balanceado y que la capacidad aeróbica de estos deportistas fue alta en relación a la población general, pero intermedia en comparación a la de deportistas. Los surfistas de la muestra no presentan un gran desarrollo de la fuerza y fuerza-explosiva de los miembros inferiores, pero poseen un buen control postural.

PALABRAS CLAVE: Surf; Somatotipo; Control postural; Salto vertical jump; $\mathrm{VO}_{2}$ Max.

\section{INTRODUCCIÓN}

El surf se originó a fines del siglo XVll en las islas de la Polinesia, principalmente en Hawai, con motivos religiosos, sociales y culturales. A principios del siglo XX el surf fue considerado como una disciplina deportiva, gracias al hawaiano, Duke Kahanawoku, quien se encargó de difundirlo por Australia, Estados Unidos y Europa (Finney \& Houston, 1996). El surf es considerado un deporte de alto riesgo y es uno de los que más se ha popularizado en los últimos años. Se calcula que en el 2012 había 35 millones de surfistas en todo el mundo de los cuales 12,5 residen en América. Se estima que para el 2020 el número de surfistas alcanzará los 47 millones (Kelly Slater Wave Co., 2012). En Chile cada año son más las personas que se suman a la práctica de este deporte. A pesar de la gran población adepta a la práctica de esta disciplina, son escasos los antecedentes publicados acerca de las características antropométricas y físicas de quienes la practican a un nivel profesional y amateur. En la literatura no existe una clara descripción de las cualidades físicas requeridas para potenciar el rendimiento deportivo o minimizar el riesgo de lesiones musculoesqueléticas. Posiblemente las cualidades físicas de estos deportistas se desarrollan en conjunto con los años de práctica, por ello los surfistas experimentados podrían tener las cualidades físicas óptimas para este deporte.

Por otro lado, las características hidrográficas de una región en particular podrían tener cierta influencia en el desarrollo y diferenciación de las cualidades físicas de los surfistas. Conocer las características antropométricas y físicas de una muestra de surfistas experimentados, podría contribuir por un lado, al conocimiento científico de este deporte, y por otro, al diseño de programas de entrenamiento y rehabilitación asociados a la práctica de este.

El objetivo de este estudio fue describir las características antropométricas y físicas de una muestra de surfistas experimentados chilenos. 


\section{MATERIAL Y MÉTODO}

El diseño utilizado en esta investigación correspondió a uno observacional descriptivo transversal.

Voluntarios. El reclutamiento de los voluntarios se realizó en primera instancia mediante una llamada telefónica en la cual se les invitó a participar en el estudio, se explicó el objetivo de este y los procedimientos involucrados. Los voluntarios que mostraron interés en participar, fueron citados al laboratorio Integrativo de Biomecánica y Fisiología del Esfuerzo (LIBFE) de la Escuela de Kinesiología de la Universidad de los Andes, donde se les explicó con mayor detalle el propósito y procedimientos del estudio. Luego de aclarar todas sus dudas, a los voluntarios se les solicitó dar su consentimiento de manera escrita. A quienes accedieron a ello, se les aplicó una encuesta con el objetivo de pesquisar los siguientes criterios de exclusión: i) Tiempo de práctica de surf menor a cinco años. ii) Frecuencia de práctica menor a una vez por semana. iii) Tabaquismo $\geq 1 / 3$ paquete/año. iv) Enfermedad cardiopulmonar. v) Lesión de los miembros inferiores o tronco en los últimos 6 meses. vi) Trastornos vestibulares centrales o periféricos. vii) Consumo de neurolépticos y/o psicotrópicos. viii) Consumo de alcohol y/o drogas en las 48 h precedentes a la evaluación.

Del total de personas reclutadas se obtuvo una muestra de 15 voluntarios surfistas experimentados chilenos $(\mathrm{n}=$ 15). Todos los procedimientos de este estudio fueron aprobados por el Comité de Bioética de la Facultad de Medicina de la Universidad de Los Andes, Chile.

Evaluaciones. Las mediciones fueron realizadas en el mismo orden que son descritas a continuación:

Somatotipo: Fue evaluado mediante el cálculo de sus tres componentes (endomorfismo, mesomorfismo y ectomorfismo) según los descrito por la International Society for the Advancement of Kinanthropometry (ISAK) (Stewart et al., 2011), para ello se midieron las siguientes variables antropométricas: circunferencia del brazo flexionado (CBF), circunferencia de pierna (CP), pliegue de tríceps (PT), pliegue subescapular (PSBE), pliegue supraespinal (PSPE), pliegue de pierna (PP), diámetro biepicondíleo del húmero $(\mathrm{DBH})$ y diámetro bicondíleo del fémur (DBF). Los componentes de endomorfismo, mesomorfismo y ectomorfismo fueron calculados de la siguiente forma (Carter, 1999):

Endomorfismo

$=-0,7182+0,1451 \cdot(\mathrm{SPC})-0,00068 \cdot\left(\mathrm{SPC}^{2}\right)+0,0000014 \cdot\left(\mathrm{SPC}^{3}\right)$

Mesomorfismo

$=0,858 \cdot(\mathrm{DBH})+0,601 \cdot(\mathrm{DBF})+0,188 \cdot(\mathrm{CCB})+0,161 \cdot(\mathrm{PCP})-0,131$

o [estatura $(\mathrm{cm})]+4,5$
Ectomorfismo

$=(\mathrm{IP}) \cdot 0,732-28,58 \quad$ Sí, IP $>40,75 \leq$

$=(\mathrm{IP}) \cdot 0,463-17,63 \quad$ Sí, $40,74<\mathrm{IP}>38,28$

$=0,10 \quad$ Sí, IP $\leq 38,28$

Donde,

$\mathrm{SPC}=(\mathrm{PT}+\mathrm{PSBE}+\mathrm{PSPE}) \cdot 170,18 /$ estatura $(\mathrm{cm})$

$\mathrm{CCB}=(\mathrm{CBF}-\mathrm{PT}) / 10$

$\mathrm{PCP}=(\mathrm{CP}-\mathrm{PP}) / 10$

$\mathrm{IP}=$ estatura $(\mathrm{cm}) /$ peso $^{1 / 3}$

Control postural: Este fue valorado mediante el desplazamiento del centro de presión (CdP), registrado por una plataforma de fuerza (FP4060-05-PT, Bertec Corp. Columbus, Oh. USA, frecuencia de muestreo de $100 \mathrm{~Hz}$ ). Los voluntarios debieron pararse descalzos sobre la plataforma por 30 $\mathrm{s}$, adoptando una postura erguida con sus pies separados, los brazos a los costados y la vista fija al frente. El control postural fue evaluado mediante la velocidad del CdP, y él área de una elipse que contuvo el $95 \%$ de las posiciones del CdP (Duarte et al., 2000).

Evaluación de Saltabilidad: Se evaluaron dos tipos de saltos que son parte de la prueba de Bosco (Bosco et al., 1983): Squat Jump (SJ): Salto vertical realizado con las manos en la cintura y desde una posición estática de triple flexión de los miembros inferiores con las rodillas en $90^{\circ}$. Su objetivo fue valorar la fuerza explosiva. Counter Movement Jump $(\mathrm{CMJ})$ : Salto vertical realizado con las manos en la cintura y desde una posición erguida y bípeda, seguida de una rápida flexo-extensión de los miembros inferiores. Este salto buscó valorar la fuerza explosiva y elástica.

Todos los saltos fueron realizados sobre una plataforma de fuerza (FP4060-05-PT, Bertec Corp. Columbus, Oh. USA, frecuencia de muestreo de $100 \mathrm{~Hz}$ ). Se solicitaron tres repeticiones de cada salto, el mejor registro de altura para cada uno fue considerado para el análisis. Las alturas fueron determinadas a partir de los tiempos de vuelo. Para ello se utilizó la siguiente fórmula (Bosco et al.):

$$
h=\mathrm{gt} / 8
$$

Donde, $h$ es la altura del salto, g la aceleración de gravedad y t el tiempo de vuelo.

Consumo máximo de oxígeno: Se realizó una prueba incremental sobre una cinta rodante en una relación de incremento de velocidad de $1 \mathrm{~km} / \mathrm{h}$ cada un minuto, hasta la fatiga. Durante esta prueba se registró de manera directa el consumo máximo de oxígeno, el cual fue expresado en $(\mathrm{ml} \cdot \mathrm{kg}) / \mathrm{min}$. Para ello se utilizó un equipo ergoespirométrico (Oxycon Mobile, Jeager \& CareFusion, Alemania). 
Análisis estadístico. Se estudió el tipo de distribución de las variables registradas mediante una prueba de ShapiroWilk. Se realizó una estadística descriptiva de las variables estudiadas, utilizando las medidas de centralización y dispersión más adecuadas para el tipo de distribución obtenida. Todos los análisis estadísticos fueron realizados usando un software estadístico (STATA/SE 12.1. StataCorp. College Station. USA). Se usó un nivel de confianza de un $95 \%(a l f a=0,05)$.

\section{RESULTADOS}

Todas las variables registradas demostraron tener una distribución normal ( $p>0,05)$, por ello se seleccionó la media y desviación estándar, como medidas de centralización y dispersión, respectivamente. Las Tablas I y II, muestran las variables registradas en cada uno de los voluntarios. En dos de los participantes, no fue posible medir el $\mathrm{VO}_{2}$ Max por razones técnicas del instrumental (Tabla II). El somatotipo de la muestra fue graficado en la somatotocarta (Fig. 1).

Tabla I. Datos demográficos y componentes somatotípicos de la muestra $(\mathrm{n}=15)$.

\begin{tabular}{lcccccc}
\hline Surfista & $\begin{array}{c}\text { Edad } \\
\text { (años) }\end{array}$ & $\begin{array}{c}\text { Peso } \\
(\mathbf{k g})\end{array}$ & $\begin{array}{c}\text { Talla } \\
(\mathbf{m})\end{array}$ & Endomorfo & Mesomorfo & Ectomorfo \\
\hline $\mathbf{1}$ & 31 & 67,0 & 1,74 & 1,84 & 4,65 & 2,25 \\
$\mathbf{2}$ & 37 & 65,1 & 1,73 & 2,68 & 3,57 & 2,92 \\
$\mathbf{3}$ & 25 & 75,6 & 1,83 & 1,98 & 4,77 & 3,10 \\
$\mathbf{4}$ & 22 & 88,9 & 1,89 & 3,41 & 5,62 & 2,42 \\
$\mathbf{5}$ & 26 & 64,3 & 1,71 & 2,61 & 3,82 & 2,66 \\
$\mathbf{6}$ & 25 & 76,0 & 1,75 & 2,09 & 4,23 & 1,66 \\
$\mathbf{7}$ & 28 & 77,0 & 1,80 & 2,57 & 4,34 & 2,39 \\
$\mathbf{8}$ & 35 & 79,0 & 1,73 & 3,53 & 5,32 & 1,04 \\
$\mathbf{9}$ & 35 & 74,0 & 1,72 & 3,65 & 4,01 & 1,41 \\
$\mathbf{1 0}$ & 19 & 75,0 & 1,82 & 1,99 & 4,83 & 3,01 \\
$\mathbf{1 1}$ & 28 & 79,0 & 1,77 & 2,93 & 5,69 & 1,62 \\
$\mathbf{1 2}$ & 23 & 68,0 & 1,77 & 2,72 & 4,37 & 3,16 \\
$\mathbf{1 3}$ & 23 & 73,5 & 1,80 & 2,67 & 4,35 & 2,87 \\
$\mathbf{1 4}$ & 23 & 70,6 & 1,78 & 2,92 & 4,72 & 2,95 \\
$\mathbf{1 5}$ & 26 & 77,8 & 1,78 & 3,02 & 5,95 & 1,94 \\
Media & 27,07 & 74,05 & 1,77 & 2,70 & 4,60 & 2,30 \\
DE & 5,20 & 6,30 & 0,04 & 0,50 & 0,70 & 0,60 \\
\hline
\end{tabular}

$\mathrm{DE}=$ Desviación estándar.

Tabla II. Datos de las variables consumo máximo de oxígeno, saltos y balance $(\mathrm{n}=15)$.

\begin{tabular}{lccccc}
\hline Surfista & $\begin{array}{c}\text { VO } \mathbf{M} \mathbf{M a} \\
(\mathbf{m L} \cdot \mathbf{m i n} / \mathbf{k g})\end{array}$ & $\begin{array}{c}\text { SJ } \\
(\mathbf{c m})\end{array}$ & $\begin{array}{c}\text { CMJ } \\
(\mathbf{c m})\end{array}$ & $\begin{array}{c}\text { Velocidad CdP } \\
(\mathbf{m m} / \mathbf{s})\end{array}$ & $\begin{array}{c}\text { Área CdP } \\
\left(\mathbf{m m}^{2}\right)\end{array}$ \\
\hline $\mathbf{1}$ & 46,4 & 23,9 & 32,4 & 7,4 & 69,3 \\
$\mathbf{2}$ & 46,4 & 29,4 & 30,4 & 5,6 & 68,1 \\
$\mathbf{3}$ & 40,5 & 31,9 & 31,9 & 7,2 & 80,6 \\
$\mathbf{4}$ & 38,3 & 27,3 & 32,4 & 8,7 & 105,8 \\
$\mathbf{5}$ & 52,0 & 35,5 & 37,3 & 7,3 & 74,5 \\
$\mathbf{6}$ & -- & 34,4 & 35,7 & 5,7 & 60,9 \\
$\mathbf{7}$ & --- & 27,5 & 29,2 & 7,8 & 66,2 \\
$\mathbf{8}$ & 38,7 & 29,4 & 32,9 & 8,5 & 50,3 \\
$\mathbf{9}$ & 44,6 & 33,1 & 35,5 & 5,8 & 61,8 \\
$\mathbf{1 0}$ & 54,6 & 36,0 & 34,2 & 9,0 & 45,4 \\
$\mathbf{1 1}$ & 50,4 & 37,3 & 40,4 & 5,0 & 40,9 \\
$\mathbf{1 2}$ & 48,1 & 32,6 & 34,4 & 7,9 & 48,6 \\
$\mathbf{1 3}$ & 55,7 & 40,9 & 42,9 & 8,6 & 77,3 \\
$\mathbf{1 4}$ & 46,9 & 36,3 & 39,0 & 6,4 & 99,3 \\
$\mathbf{1 5}$ & 51,2 & 43,8 & 48,6 & 8,5 & 68,1 \\
Media & 47,2 & 33,2 & 35,8 & 7,2 & 18,4 \\
DE & 5,6 & 5,3 & 5,1 & 1,3 & \\
\hline
\end{tabular}

$\mathrm{DE}=$ desviación estándar, $\mathrm{VO}_{2}$ max= consumo máximo de oxígeno, $\mathrm{SJ}=$ Squat Jump, CMJ=Counter Movement Jump. $\mathrm{CdP}=$ Centro de presión. Velocidad $\mathrm{CdP}=$ velocidad del desplazamiento del centro de presión. Área $\mathrm{CdP}=$ área de desplazamiento del centro de presión. 


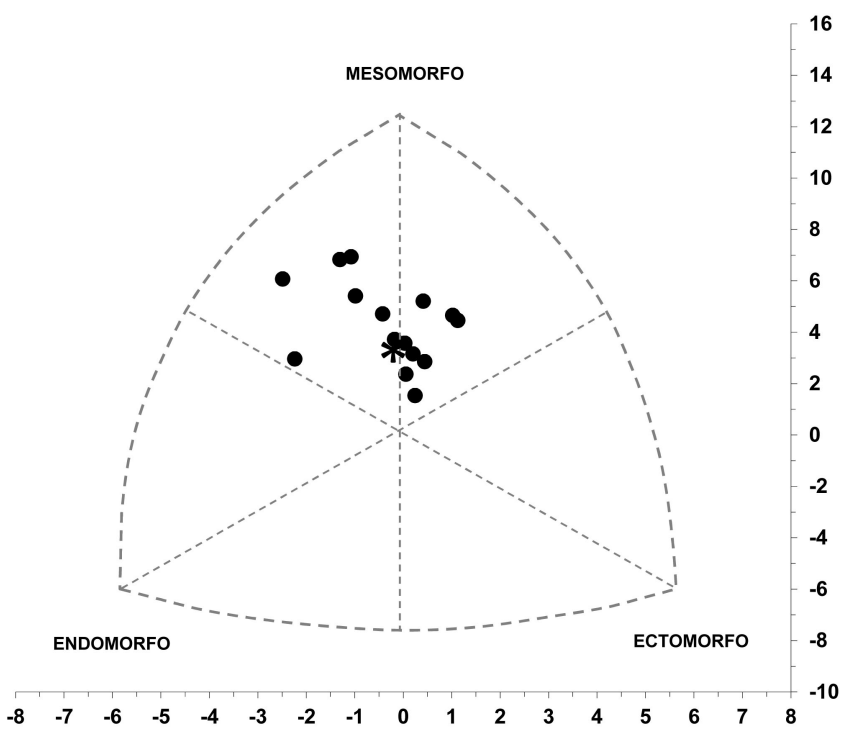

Fig. 1. Distribución del somatotipo de los surfistas de la muestra $(n=15)$ en la somatocarta. El asterisco indica el promedio de toda la muestra.

\section{DISCUSIÓN}

Para enfrentar las demandas del océano, los surfistas deben responder a extensos períodos de ejercicio, utilizando tanto sus miembros superiores como inferiores, es una actividad de intensidad y duración variable, que incluye períodos de recuperación activos. La exigencia física durante la práctica puede variar desde 20 min hasta 4 ó 5 h cuando existen buenas condiciones de oleaje. Habitualmente los surfistas pasan horas en el agua y muchas veces estas sesiones son vistas como un pasatiempo y no como una práctica deportiva, por lo que la mayoría de los surfistas no tienen conciencia del nivel de entrenamiento que demanda realizar de manera segura este deporte. El surf es, ante todo, un deporte que requiere habilidades excepcionales con respecto a las cualidades físicas, técnicas y psicológicas (Lowdon et al., 1989).

Antropometría. Antecedentes previos indican que los surfistas en general no presentan altas tallas. Los surfistas de competición parecen ser más pequeños y livianos que el promedio de la población de deportistas de la misma edad (Mendez-Villanueva \& Bishop, 2005). El promedio de altura de los mejores 44 surfistas hombres del ranking ASP (Association of Surfing Professionals, pertenecientes al World Championship Tour 2003) es de 174,7 $\pm 6,1$ cm (161-188 cm) (Mendez-Villanueva et al., 2003). Estos valores son similares a los encontrados en el presente estudio (177 \pm 4 $\mathrm{cm})$. Si comparamos esta altura promedio con otros deportistas acuáticos, se puede observar que los surfistas son notablemente más bajos que, por ejemplo, los nadadores de elite $(183,8 \pm 7,1$ $\mathrm{cm} ; \mathrm{n}=231)$ y jugadores de waterpolo $(186,5 \pm 6,5 \mathrm{~cm} ; \mathrm{n}=190)$ (Carter \& Ackland, 1994). Una razón de esta menor talla, podría ser una de aspecto mecánico. La estabilidad de un cuerpo es inversamente proporcional a su altura (Hayes, 1982). El surf es un deporte que requiere de gran estabilidad, por ello una baja estatura podría ser una ventaja en su práctica. Con respecto a la masa corporal, se ha repor-

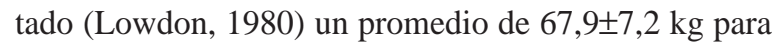
76 surfistas hombres de nivel internacional, este valor es inferior al encontrado en nuestro estudio $(74 \pm 6,3 \mathrm{~kg}) . \mathrm{Si}$ bien es un poco más alto que el promedio de profesionales extranjeros, existe aún una mayor diferencia si se compara con otros deportistas acuáticos de elite (Carter \& Ackland), quienes presentan un promedio de $78 \pm 7 \mathrm{~kg}$ para los nadadores y $86 \pm 8,4 \mathrm{~kg}$ para los waterpolistas. Por lo tanto, se podría afirmar que el perfil del surfista, se acerca a un cuerpo relativamente pequeño y ligero, el cual resultaría ser ventajoso para la realización de movimientos específicos sobre la tabla de surf. Sin embargo, existen otros estudios que contradicen esta postura y señalan que "la composición corporal no parece jugar un rol importante en el rendimiento del surf" (MendezVillanueva \& Bishop).

El somatotipo es una de varias técnicas para representar la morfología del cuerpo humano. Lowdon analizó el somatotipo de 76 hombres surfistas profesionales a nivel internacional y reportó que estos surfistas tuvieron un somatotipo mesomorfo balanceado (endomorfismo: 2,6; mesomorfismo: 5,2 y ectomorfismo: 2,6). El somatotipo de la muestra evaluada en el presente estudio también correspondió a uno mesomorfo balanceado (endormorfismo: 2,7, mesomorfismo: 4,6 y el ectomorfismo: 2,3). A pesar de la similitud de los somatotipos de ambas muestras, en la nuestra se observó un menor componente mesomórfico, lo cual puede ser interpretado como una menor cantidad de tejido muscular, esto seguramente está relacionado con los hábitos de entrenamiento de estos deportistas. Un entrenamiento sistemático de fuerza, puede aumentar el componente mesomórfico. Seguramente los surfistas de nivel internacional, dentro de sus hábitos deben tener incorporada rutinas de entrenamientos de fuerza, tales como el uso de pesas, lo cual sería el responsable del mayor componente mesomórfico en relación a la muestra del presente estudio.

Control Postural. La estabilidad postural tiene por objetivo mantener una postura estable, lo cual mecánicamente se traduce en conservar proyectado el centro de masa dentro de la base de soporte. En el presente estudio esta habilidad fue valorada mediante el desplazamiento del centro de presión (CdP). Se ha demostrado que la velocidad y área descrita por el CdP, son buenos indicadores de la estabilidad postural (Du Pasquier et al., 
2003). El control postural de la muestra evaluada, es muy similar al descrito en otras muestras de surfistas. De este modo la velocidad del CdP registrada en el presente estudio $(7,3$ $\mathrm{mm} / \mathrm{s})$, fue parecida a la registrada por Paillard et al. (2011) $(8 \mathrm{~mm} / \mathrm{s})$, y algo más alta que la informada por Frank et al. (2009) $(4 \mathrm{~mm} / \mathrm{s})$. Por otro lado, el área del centro de presión registrada en la presente muestra $\left(68,1 \mathrm{~mm}^{2}\right)$, fue menor que la registrada por Paillard et al. $\left(125 \mathrm{~mm}^{2}\right)$. En base a los resultados obtenidos se observó que los surfistas de la muestra evaluada poseen un buen control postural, muy similar al reportado en otras muestras de surfistas.

Saltabilidad. En la mayoría de los deportes, la fuerza muscular es uno de los factores relacionados con el rendimiento y el riesgo de sufrir lesiones musculoesqueléticas. Para un adecuado diseño de un programa de entrenamiento es necesario conocer los requerimientos de fuerza del deporte específico y los niveles que presenta cada deportista en particular. En este contexto la saltabilidad permite de manera accesible y sencilla evaluar funcionalmente la fuerza y potencia de los miembros inferiores. Estas dos últimas cualidades han sido reconocidas como factores determinantes del rendimiento en el surf (Mendez-Villanueva \& Bishop). En el presente estudio se midieron dos tipos diferentes de saltos, el SJ que evalúa la fuerza explosiva y el CMJ, que aparte de valorar la fuerza explosiva también mide la fuerza elástica. De manera particular, en la literatura no se disponen de datos de saltabilidad en muestras de surfistas. La altura alcanzada por la fuerza explosiva de la muestra evaluada (SJ $33,29 \pm 5 \mathrm{~cm}$ ) resultó ser menor que en otros deportes, tales como futbolistas (SJ 37,8 cm) (Malliou et al., 2003) y jugadores de baloncesto $(37,9 \mathrm{~cm})$ (Köklü et al., 2011). La misma tendencia fue observada al comparar las alturas alcanzadas por la fuerza elástico-explosiva, en futbolistas $(39,2 \mathrm{~cm})$ (Malliou et al.), basquetbolistas $(40,6 \mathrm{~cm}$ ) (Köklü et al.) y en los surfistas del presente estudio $(35,81 \pm 5,1 \mathrm{~cm})$. Los valores registrados en la muestra evaluada, podrían indicar que los requerimientos de fuerza y potencia musculares no son altos para el surf. Esto puede deberse a las características ambientales que implica la práctica de este deporte. Al desarrollarse en un ambiente acuático, las fuerza de reacción que aplica el agua a la tabla y esta última a los deportistas, no debe ser de una alta magnitud, dada la naturaleza deformable del agua. De esta manera los niveles de fuerza y potencia muscular deben ir direccionados más a mantener la estabilidad postural sobre la tabla, que a resistir grandes fuerzas de impacto.

Consumo Máximo de Oxígeno. Una de las actividades específicas que conlleva la práctica del surf es el "remo", esta consiste en una forma adaptada del nado, la cual se realiza tendido en prono sobre la tabla de surf, impulsándose principalmente con los miembros superiores. Esta es una actividad predominantemente aeróbica, la cual representa de manera intermitente, la mayor parte del tiempo de práctica del surf (Meir et al., 1991; Felder et al., 1998; Mendez-Villanueva et al.). Mier et al. describieron que en una hora de surf recreacional, la frecuencia cardiaca promedio equivale al 80 $\%$ de la frecuencia cardiaca máxima (FC max). Esto indica que la capacidad aeróbica es una cualidad relevante en la práctica de este deporte. La capacidad aeróbica de un deportista puede ser valorada mediante la evaluación del $\mathrm{VO}_{2} \mathrm{Max}$, realizada mediante pruebas de esfuerzos progresivos sobre máquinas trotadoras o bicicletas ergométricas, en el caso particular del surf, podría ser evaluado utilizando algún tipo de ergométro de brazos. Sawka (1986) evaluó una muestra de surfistas en dos condiciones diferentes: una en posición prona con el uso de miembros superior y la otra corriendo sobre una trotadora. Compararon los $\mathrm{VO}_{2}$ max y concluyeron que los valores del $\mathrm{VO}_{2}$ max en prono equivalen sólo al $70 \%$ de los valores obtenidos durante el trote. Estas diferencias fueron atribuidas a la mayor masa muscular involucrada en la acción de correr en comparación a la de remar. En el presente estudio se decidió valorar la capacidad aeróbica durante la carrera en cinta rodante, dado que esta prueba revela la real capacidad aérobica de los deportistas. Mier et al., evaluaron la capacidad aeróbica de surfistas $(\mathrm{n}=12)$ utilizando una cinta rodante, obtuvieron un $\mathrm{VO}_{2}$ max de $56,3 \pm 3,9(\mathrm{~mL} \cdot \mathrm{min}) / \mathrm{kg}$, valor que superó en aproximadamente $10 \mathrm{ml} / \mathrm{min} / \mathrm{kg}$ al promedio de la muestra evaluada por nosotros $(47,2(\mathrm{~mL} \cdot \mathrm{min}) / \mathrm{kg})$. Esto indica que la capacidad aeróbica de los surfistas de la muestra evaluada es menor que surfistas de nivel internacional, esto también podría ser atribuido a la falta de entrenamientos específicos de la capacidad aeróbica en la rutina de los deportistas que conformaron la muestra del presente estudio.

Una de las principales limitantes del presente estudio es que la muestra obtenida no tiene una base poblacional de los surfistas chilenos, por lo cual, la validez externa de este estudio es limitada. Por otro lado, en el presente estudio no se planteó alguna evaluación funcional de los miembros superiores, las cuales tienen un rol importante en la práctica de este deporte.

\section{CONCLUSIÓN}

En este estudio se describieron las características antropométricas y físicas de una muestra de surfistas experimentados chilenos, esta descripción reveló que el somatotipo de la muestra fue mesomórfico balanceado, que la capacidad aeróbica de estos deportistas fue alta en relación a la población general, pero intermedia en relación a la de deportistas. Los surfistas de la muestra no presentan un gran desarrollo de la fuerza y fuerza-explosiva de los miembros inferiores, y poseen un buen control postural. 
VALDÉS, V. M. I. \& GUZMÁN-VENEGAS, R. Description of somatotype and physical qualities of experienced chilean men surfers. Int. J. Morphol., 34(1):23-28, 2016.

SUMMARY: Surfing is a sport that has been growing rapidly in popularity throughout Chile. However, there are very few studies that describe the anthropometric and physical characteristics of these athletes in this country. The objective of this study was to describe anthropometric and physical characteristics in Chilean surfers. The study design is a descriptive observational cross study. Somatotype, postural stability, Squat Jump (SJ) and Counter Movement Jump (CMJ) and maximal oxygen uptake $\left(\mathrm{VO}_{2} \max \right)$ were evaluated in 15 experienced, Chilean surfers (age: 27.1 \pm 5.2 ; height: $1.77 \pm 0.04$; weight: $74.1 \pm 6.3$ ). Somatotype sample average was: endomorphism: $2.7 \pm 0.5$, mesomorphism: $4.6 \pm 0.7$ and ectomorphism: $2.3 \pm 0.6$. In postural stability test, the average of the center of pressure displacement of speed and area was $7.2 \pm 1.3 \mathrm{~mm} / \mathrm{sec}$ and $68.1 \pm 18.4 \mathrm{~mm}^{2}$ respectively. The height average of the jumps was $33.2 \pm 5.3 \mathrm{~cm}$ for $\mathrm{SJ}$ and $35.8 \pm 5.1 \mathrm{~cm}$ for CMJ. The average of $\mathrm{VO}_{2} \max$ reached $47.2 \pm 5.6(\mathrm{~mL} \cdot \mathrm{min}) / \mathrm{kg}$. This description reflects the sample's somatotype was balanced mesomorph, their aerobic capacity was highlevel in relation to the general population, but is middle level in comparison to other athletes. Surfers of this sample didn't show a large force development or explosive strength in their lower limbs and hold a good postural control.

KEY WORDS: Surf; Somatotype; Postural control; Vertical jump; $\mathrm{VO}_{2}$ max.

\section{REFERENCIAS BIBLIOGRÁFICAS}

Bosco, C.; Luhtanen, P. \& Komi, P. V. A simple method for measurement of mechanical power in jumping. Eur. J. Appl. Physiol. Occup. Physiol., 50(2):273-82, 1983.

Carter, J. E. L. The Heath-Carter Anthropometric Somatotype. Instruction Manual. San Diego, San Diego State University, 1999.

Carter, J. E. L. \& Ackland, T. R. Kinanthropometry in Aquatic Sports. A Study of World Class Athletes. Champaign, Human Kinetics, 1994.

Du Pasquier, R. A.; Blanc, Y.; Sinnreich, M.; Landis, T.; Burkhard, P. \& Vingerhoets, F. J. The effect of aging on postural stability: a cross sectional and longitudinal study. Neurophysiol. Clin., 33(5):213-8, 2003.

Duarte, M.; Harvey, W. \& Zatsiorsky, V. M. Stabilographic analysis of unconstrained standing. Ergonomics, 43(11):1824-39, 2000.

Felder, J. M.; Burke, L. M.; Lowdon, B. J.; Cameron-Smith, D. \& Collier, G. R. Nutritional practices of elite female surfers during training and competition. Int. J. Sport Nutr., 8(1):36-48, 1998.

Finney, B. R. \& Houston, J. D. Surfing: A History of the Ancient Hawaiian Sport. San Francisco, Pomegranate Artbooks, 1996.

Frank, M.; Zhou, S.; Bezerra, P. \& Crowley, Z. Effects of long-term recreational surfing on control of force and posture in older surfers: a preliminary investigation. J. Exerc. Sci. Fit., 7(1):31-8, 2009.
Hayes, K. C. Biomechanics of postural control. Exerc. Sport Sci. Rev., 10:363-91, 1982.

Kelly Slater Wave Co. Página de internet. Kelly Slater Wave Co., 2012. Available from: http://www.kswaveco.com

Köklü, Y.; Alemdaroglu, U.; Koçak, F. Ü.; Erol, A. E. \& Fındıkoglu, G. Comparison of Chosen Physical Fitness Characteristics of Turkish Professional Basketball Players by Division and Playing Position. Comparison of chosen physical fitness characteristics of Turkish professional basketball players by division and playing position. J. Hum. Kinet., 30:99-106, 2011.

Lowdon, B. J. The somatotype of international surfboard riders. Aust. J. Sci. Med. Sport, 12:34-9, 1980.

Lowdon, B. J.; Bedi, J. F. \& Horvath, S. M. Specificity of aerobic fitness testing of surfers. Aust. J. Sci. Med. Sport, 21:7-10, 1989.

Meir, R. A.; Lowdon, B. J. \& Davie, A. J. Heart rates and estimated energy expenditure during recreational surfing. Aust. J. Sci. Med. Sport, 23:70-4, 1991.

Mendez-Villanueva, A. \& Bishop, D. Physiological aspects of surfboard riding performance. Sports Med., 35(1):55-70, 2005.

Mendez-Villanueva, J. A.; Bishop, D. \& Hamer, P. Activity patterns of elite surfing competition. J. Sci. Med. Sport, 4 (Suppl.):11, 2003.

Malliou, P.; Ispirlidis, I.; Beneka, A.; Taxildaris, K. \& Godolias, G. Vertical jump and knee extensors isokinetic performance in professional soccer players related to the phase of the training period. Isokinet. Exerc. Sci., 11(3):165-9, 2003.

Paillard, T.; Margnes, E.; Portet, M. \& Breucq, A. Postural ability reflects the athletic skill level of surfers. Eur. J. Appl. Physiol., 111(8):1619-23, 2011.

Sawka, M. N. Physiology of upper body exercise. Exerc. Sport Sci. Rev., 14:175-211, 1986.

Stewart, A.; Marfell-Jones, M.; Olds, T. \& de Ridder, H. International Standards for Anthropometric Assessment. Lower Hutt, ISAK, 2011.

Dirección para Correspondencia:

MSc. Rodrigo Guzmán-Venegas

Lab. Integrativo de Biomecánica y Fisiología del Esfuerzo

Universidad de los Andes

Avda. Monseñor Álvaro del Portillo 12455

Las Condes

Santiago

CHILE

Email: rguzman@uandes.cl 